\title{
BMJ Open Protocol for a pilot, randomised, double- blinded, placebo-controlled trial of prophylactic use of tranexamic acid for preventing postpartum haemorrhage (TAPPH-1)
}

To cite: Alam A, Bopardikar A, Au S, et al. Protocol for a pilot, randomised, doubleblinded, placebo-controlled trial of prophylactic use of tranexamic acid for preventing postpartum haemorrhage (TAPPH-1). BMJ Open 2017;7:e018586. doi:10.1136/ bmjopen-2017-018586

- Prepublication history for this paper is available online. To view these files, please visit the journal online (http://dx.doi. org/10.1136/bmjopen-2017018586).

Received 7 July 2017

Revised 31 August 2017

Accepted 22 September 2017

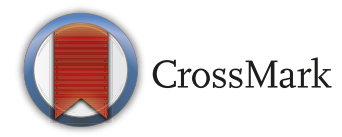

For numbered affiliations see end of article.

Correspondence to

Dr Asim Alam;

asim.alam@sunnybrook.ca

\section{ABSTRACT}

Introduction Postpartum haemorrhage (PPH) is the leading cause of maternal morbidity and mortality worldwide. Despite the availability of multiple uterotonic agents, the incidence of PPH continues to rise. Tranexamic acid (TXA) has been shown to be a safe, effective and inexpensive therapeutic option for the treatment of PPH, however, its use prophylactically in mitigating the risk of PPH is unknown. This pragmatic randomised prospective trial assesses the feasibility and safety of administering TXA at the time of delivery for the prevention of PPH. Methods and analysis A pilot pragmatic randomised double-blinded placebo-controlled trial will be performed. 58 singleton parturients at term $>32$ weeks, undergoing either spontaneous vaginal delivery, or caesarean section will be randomised to receive $1 \mathrm{~g}$ of TXA or placebo $(0.9 \%$ saline) intravenously. The primary outcome assessed will be the feasibility of administrating TXA, along with collecting data regarding safety of drug administration. The groups will also be analysed on efficacy of mitigating the onset of PPH and clinically relevant variables. Demographic, feasibility, safety and clinical endpoints will be summarised and the appropriate measures of central tendency and dispersion will be presented.

Ethics and dissemination This protocol was approved by the Sunnybrook Health Sciences Centre Research Ethics Board (number: 418-2016). The results will be disseminated in a peer-reviewed journal and at scientific meetings.

Trial registration number NCT03069859; Pre-results.

\section{INTRODUCTION}

Postpartum haemorrhage (PPH) is the leading cause of maternal morbidity and mortality worldwide. Clinically, PPH is defined as blood loss greater than $500 \mathrm{~mL}$ after spontaneous vaginal delivery (SVD) or greater than $1000 \mathrm{~mL}$ after caesarean section (CS). ${ }^{1}$ Although a number of treatments exist, severe haemorrhage and subsequent maternal morbidity and mortality are an ongoing concern, particularly in low-income

\section{Strengths and limitations of this study}

- Clinically important question that is centred around patient care.

- Protocolised care will not be instituted.

- Pragmatic trial applicable to parturients undergoing spontaneous vaginal delivery or caesarean section.

- Clinical effect size will not be adequately captured in this pilot study.

- Study conducted in a high-resourced quaternary care centre may not be as generalisable to lowerrisk centres with less obstetrical support.

countries. $^{2}$ There is a demand for a safe, low-cost, effective therapy that can be administered in a wide range of healthcare settings to reduce the incidence of $\mathrm{PPH}{ }^{3}$

The worldwide incidence of PPH is estimated to be between $6 \%$ and $11 \%$ with substantial regional variability. ${ }^{45}$ The incidence of PPH is greatest in low/middle-income nations, particularly in sub-Saharan Africa where it can be larger than $30 \% .^{245}$ Yet, developed nations are experiencing an increasing rate of $\mathrm{PPH}$ in comparison to low/ middle-income nations. ${ }^{6-10}$ The apparent causes for this increase are still not fully understood. ${ }^{11}$ In the USA, PPH has increased 26\% between 1994 and 2006 from 2.3\% to $2.9 \% .{ }^{10}$ In Canada, PPH rates have increased $22 \%$ between 2003 and $2010 .{ }^{9}$ In particular, the incidence of $\mathrm{PPH}$ that requires blood transfusion has increased from 36.7 to 50.4 cases per 10000 deliveries in the same time period. ${ }^{9}$ Similarly, $\mathrm{PPH}$ resulting in hysterectomy has increased from $4.9 \%$ to $5.8 \%{ }^{9}$ Following these trends, the overall severe maternal morbidity rate between 2003 and 2011 has increased from 14.2 to 15.4 cases per 1000 deliveries in Canada. ${ }^{12}$ 
$\mathrm{PPH}$, blood transfusion and hysterectomy are principal causes of maternal morbidity-all potentially inter-related outcomes. ${ }^{12}$ Although maternal mortality is relatively rare in developed nations, PPH may be implicated in up to $27 \%$ of all cases. ${ }^{13}$

\section{Economic burden of PPH}

Unfortunately, there is a paucity of published data on the true economic impact of maternal haemorrhage. However, as PPH, blood transfusion and hysterectomy are all inter-related and primary drivers of maternal morbidity, even one PPH case is assumed to bequeath a substantial economic burden on society. In a recent economic analysis from the USA, the cost of PPH increased the average direct hospitalisation costs of the delivery from US $\$ 5000$ to approximately US $\$ 90000 .^{14}$ Thus, when factoring multiple potential long-term sequelae of PPH along with treatment complications, there is a significant economic impact towards healthcare costs.

In low/middle-income nations, this effect is even more evident. Morbidity and mortality rates from PPH are devastating, affecting approximately 300000 women and resulting in 72000 deaths yearly in sub-Saharan Africa alone. ${ }^{3}$ Maternal morbidity of a single individual in specific African nations was found to reduce the per capita GDP by US $\$ 0.36$ per year. ${ }^{15}$ Given the role of PPH in maternal morbidity, the economic burden on low-income and middle-income nations can be substantial.

\section{Tranexamic acid}

Tranexamic acid (TXA) is an antifibrinolytic agent commonly used to treat and/or prevent blood loss from surgery, trauma, haemophilia and heavy menstrual bleeding. TXA works by blocking the breakdown of blood clots (fibrinolysis), which prevents bleeding. It prevents the conversion of plasminogen to plasmin. ${ }^{16}$ The recommended dose of TXA for the treatment of $\mathrm{PPH}$ is $1 \mathrm{~g}$, and the cost of TXA is US\$4-6/g. ${ }^{17}$ Assuming that this intervention can produce even a modest absolute risk reduction of $1.55 \%$, the number needed to treat (NNT) or prevent one case of PPH would be 64.5. The cost of this intervention per NNT is US\$322.50. Comparatively, the direct relative average cost savings can be up to US\$85000 if one incidence of PPH is avoided. The economical application of prophylactically using TXA in this clinical scenario in North America, should it be efficacious, is therefore crucial.

\section{Evidence gaps in the prophylactic use of TXA for PPH}

TXA appears to be a suitable target for therapeutic action to help prevent the onset of PPH. In addition to being cost-effective, it has a proven safety profile and has become the drug of choice to reduce haemorrhage in numerous clinical settings. ${ }^{18-21}$ There are several small randomised controlled trials (RCTs) that have assessed whether a prophylactic loading dose of TXA can prevent haemorrhage among parturients. Three recent systematic reviews on the topic have determined that although its pre-emptive use is associated with a reduction in key bleeding outcomes in both SVD and CS, firm conclusions could not be made due to uncertainties related to study design. ${ }^{322} 23$

World Maternal Antifibrinolytic Trial (WOMAN trial), a large pragmatic international RCT, modelled after the CRASH-2 trial, was recently published to determine if TXA can reduce mortality in patients who have established $\mathrm{PPH} .{ }^{24}$ The investigators of this trial identified a significant decrease of $19 \%$ in death from bleeding in patients with established PPH. Despite this large effect size, the trial design does not inform us of whether a prophylactic dose of TXA will be effective in preventing PPH from occurring in the first place; hence, the need for this study.

The TRAnexamic Acid for Preventing postpartum hemorrhage after vaginal delivery (TRAPP trial) trial is a French RCT currently underway to assess the effect of a prophylactic dose of TXA on PPH in SVD patients. ${ }^{13}$ Unfortunately, this study has limited external validity since it only includes patients undergoing SVD, and does not capture women undergoing operative deliveries. In addition, there are many exclusion criteria, the investigators use in their study design which would make any results of this trial less generalisable. Finally, the characteristics of disease incidence are not congruent with those in found in a North American setting, and thus the sample size calculated for the study is based on much higher rates of PPH.

\section{Rationale for this pilot trial}

Preventing PPH and its subsequent sequelae is an important clinical and economic goal. The application of TXA has been well studied for the treatment of PPH, however, its role in prevention is not well understood. A recent Cochrane systematic review assessing all RCTs using TXA for the prevention of PPH was able to identify 12 studies, all of which were conducted in low-resource and middle-resource settings, with enrolment of less than 1000 parturients. ${ }^{22}$ Currently, there has not been a study in the North American setting, and thus recruitment and patient involvement in such a study protocol is unknown. Furthermore, these small-scale RCTs have significant variability in their collection of adverse effects. For example, Abdel-Aleem et al identified that $74 \%$ of patients in the TXA group had adverse reactions, whereas, a similar study intervention protocol by Yehia et al showed that adverse reactions were not experienced by any patients. ${ }^{25} 26$

An adequately powered double-blinded RCT that compares TXA to placebo is required to identify the efficacy of TXA in the prevention of PPH, however, there is currently insufficient, heterogeneous evidence to support a large-scale RCT. Thus, this pilot study is a feasibility trial to elucidate the logistical challenges of administering this intervention, and the possible pitfalls prior to devoting significant resources to execute a full-scale multicentre trial. 


\section{Hypothesis}

The administration of TXA to parturients prior to delivery is feasible and safe in highly regulated healthcare settings.

\section{Specific aims and objectives}

This double-blinded pilot RCT will determine the feasibility and safety of administering TXA to parturients undergoing both SVD and CS. The aim will be to identify challenges in recruitment, resource allocation and logistics of administering the intervention for facilitating the potential planning of a full-scale RCT. The secondary objectives of this trial will assess the impact of the intervention on specific clinical outcome measures.

\section{METHODS AND ANALYSIS Study design}

The Standard Protocol Items: Recommendations for Interventional Trials recommendations were followed in preparation for the protocol. ${ }^{27}$ This study is designed to be a prospective pragmatic pilot, single-centred randomised, double-blind, placebo-controlled trial at Sunnybrook Health Sciences Centre (SHSC). All approved amendments to this study will be communicated to relevant parties (eg, investigators, study participants, trial registries).

\section{Off-label use of TXA}

This trial involves investigating the off-label use of TXA. Health Canada approval (No Objection Letter) and SHSC Research Ethics Board (REB) approval have been obtained.

\section{Duration of study}

The recruitment phase of this study will last for 4 months, beginning January 2018.

\section{Patient recruitment and informed consent}

Study inclusion and exclusion criteria are listed in box 1 . Enrolment of patients will occur at SHSC, and study personnel will use multiple strategies to identify potentially eligible patients (ie, reviews of daily elective CS list, review of parturients admitted to the labour and delivery ward, regularly scheduled antenatal visits with obstetricians, screen daily preoperative assessment clinic lists, among others). This includes giving potential participants an information pamphlet at their clinical visits. This pamphlet will detail the background and nature of the study and contact information for individuals who are interested in enrolling in the study.

Patients will be approached to participate in the trial and then asked for informed consent and voluntary participation. Study personnel will ensure that patients understand all items outlined in the consent form prior to obtaining written consent.

\section{Randomisation and blinding}

A total of 58 patients will be randomised in this study to receive either TXA or placebo (figure 1). SHSC pharmacy

\section{Box 1 Inclusion and exclusion criteria}

Inclusion criteria
singleton pregnancy
confirmed pregnancy
greater than 18 years of age at time of consent
gestational age $>320^{0 / 7}$ weeks.
Exclusion criteria
lack of patient consent
multiple pregnancy
history of eclampsia or pre-eclampsia in current pregnancy
imminent delivery (<2 hours) from presentation suspected
history of cardiovascular complications:
- coronary artery disease or myocardial infarction
- repaired or unrepaired congenital heart disease
- vascular disease(s)
- severe unstable arrhythmia (eg, rapid atrial fibrillation,
paroxysmal fibrillation, atrial flutter, among others)
- congestive heart failure
- contraindication to tranexamic acid (TXA):
- history of venous thromboembolism
- active thromboembolic disease
- high risk of thrombosis (eg, factor V Leiden or protein C deficiency)
- acquired disturbances of colour vision
- allergy to TXA
- history of seizure disorder
- pre-existing haematuria
- history of renal insufficiency
move out of town)

will create a randomisation sequence that is stratified based on expected mode of delivery. Those randomised to the TXA group will receive $1 \mathrm{~g}$ of TXA $(10 \mathrm{~mL})$ diluted in $50 \mathrm{~mL}$ of $0.9 \%$ saline (Total $60 \mathrm{~mL}$ ), while those in the placebo group will receive $10 \mathrm{~mL}$ of $0.9 \%$ saline diluted in $50 \mathrm{~mL}$ of $0.9 \%$ saline (total $60 \mathrm{~mL}$ ) (Omega Laboratories, Montreal, Canada). The products will be identically labelled with unique numerical identifiers and will be blinded to subjects, clinicians, investigators and data collectors/analysts. Excess and waste investigational products (IPs) will be returned to the pharmacy for appropriate disposal as per Good Manufacturing Processes and logged by the pharmacy.

\section{Study intervention phase}

For participants undergoing SVD, the TXA (or placebo) will be administered on delivery of the anterior shoulder. In participants undergoing elective-CS the TXA (or placebo) will be administered when the obstetrician begins to cleanse the incision site. For patients who were planning to have SVD and then require a CS, the TXA (or placebo) that was to be given during the delivery of the anterior shoulder will instead be given as per elective-CS direction (when the obstetrician begins to cleanse the incision site). In all cases, the TXA (or placebo) will be delivered through a secondary intravenous line into the main free flowing line. 




Figure 1 Study flow chart of patients consented and randomised to TXA or placebo. CS, caesarean section; SVD, spontaneous vaginal delivery; TXA, tranexamic acid.

Aside from the intervention of the TXA (or placebo), usual obstetrical care will take place as per the obstetrician and anaesthesiologist. Protocolised care will not be instituted, as this will detract from the pragmatic generalisability of the results. However, aspects of the obstetrical/ anaesthesia care that will be important to the outcome will be collected through case report forms.

\section{Follow-up and data collection}

Study personnel will complete a detailed chart abstraction to assess intraoperative events and major post-delivery adverse events. All data will be categorised as defined by the primary and secondary outcome measures. Data collection will also occur with follow-up phone calls at 6 weeks ( \pm 14 days) and 12 weeks ( \pm 14 days) post-delivery.

All data will be stored on secured SHSC servers. Only study investigators will have access to the final trial dataset.

\section{Patient withdrawal}

Patients may choose to withdraw at any time for the duration of the study. If the participant (or the substitute decision-maker) withdraws previously given informed consent or refuses to consent for continuation in the trial, the data collected to the point of withdrawal of consent will be used as part of the intention to treat analysis. Patients may also be withdrawn at the discretion of the investigator for any safety reasons.

\section{Box 2 Secondary objectives}

incidence of postpartum haemorrhage (PPH)

incidence of severe PPH:

- requiring blood transfusion

- emergency hysterectomy

- operative haemostatic procedures

- admission to intensive care unit (ICU) \pm disseminated intravascular coagulopathy within 24 hours

- rates of blood product transfusion within 48 hours

- hospital length of stay

- postoperative complications (including thromboembolic and renal complications) at 6 and 12 weeks

- seizure within 24 hours post-delivery

- acute kidney injury during initial hospitalisation

- ICU admission during initial hospitalisation

- health status of newborn at 6 and 12 weeks

- minor adverse events (ie, nausea/vomiting, diarrhoea, dizziness, headache, light-headedness, visual disturbances or changes).

All relevant adverse events identified will be reported as required as per Health Canada and SHSC REB Regulations.

\section{Outcomes}

The primary outcome of this study is to assess feasibility of administration of the IP, and will be defined as $85 \%$ of randomised patients receiving IP. Further endpoints that will be assessed include the total time and cost required to randomise 58 patients and missed patients.

Secondary outcome measures assessing safety and clinical endpoints as described in box 2 will also be collected.

\section{Risks to the safety of study participants}

In order to ensure safety, an interim analysis will be conducted approximately at the halfway mark of randomisation. The study is a feasibility trial and not powered adequately to detect for safety endpoints. However, safety will be determined by assessing if there is a significant difference in serious adverse events experienced by patients between the two groups. The results will be reviewed by an independent Data Safety Monitoring Board to ensure that the differences between groups are not larger than expected. If a larger than expected difference is found, the study will be stopped prior to the enrolment of the full sample size.

\section{Sample size calculation}

To achieve the primary outcome of feasibility as defined by having $85 \%$ of all patients randomised receive the IP, we calculated that 58 patients will be required to give us reasonable precision in our estimate of the primary outcome (95\% CI $73.2 \%$ to $93 \%$ ).

SHSC receives about 4500 deliveries per year. Assuming a conservative recruitment rate of $5 \%$ in eligible patients, we estimate that approximately 58 patients can be randomised in a little over 3 months. Should this recruitment be found feasible (and within the allotted timeframe), we estimate that a larger multicentred RCT could 
be completed in less than 2 years if only a handful of centres from the affiliate GTA-OBS Research Network are involved. This network oversees approximately 65000 deliveries per year. Based on this, 3408 patients are required to be enrolled to have an $80 \%$ chance to detect a statistically significant decrease in the incidence of PPH, as represented by a $35 \%$ relative risk reduction.

\section{Data analyses}

For this pilot trial, statistical analysis will only be used to determine if there are gross differences between comparative groups, recognising they are not adequately powered to show a true effect. Demographic, feasibility, safety and clinical endpoints will be summarised and the appropriate measures of central tendency and dispersion will be presented (means and SDs or medians and IQRs). Categorical secondary feasibility, safety and clinical outcomes will be analysed using $\chi^{2}$ or Fisher's exact analysis. Continuous secondary feasibility and clinical outcomes will be analysed using a t-test for non-parametric continuous outcomes. For analysis of the secondary clinical endpoints, relative risk ratios and 95\% CIs with two-sided $p$ values of 0.05 will be considered significant.

\section{ETHICS AND DISSEMINATION}

\section{Safety considerations}

TXA has an excellent safety profile, yet because it inhibits fibrinolysis, there are concerns that it may increase the risk of venous and arterial thrombotic events, especially in patients with a previous history of thrombosis and or in patients with a baseline risk that is already elevated (box 3). However, a recent systematic review which examined the use of TXA among surgical patients found no significant increases in the incidence of myocardial infarction, stroke, deep vein thrombosis or pulmonary embolism. ${ }^{18}$ Similar results were also shown in a recent meta-analysis among orthopaedic surgical patients, with the caveat that most of the included studies excluded patients with a history of cardiovascular disease, thromboembolic events

\section{Box 3 Known possible reactions to tranexamic acid}

Known possible adverse events*

- gastrointestinal disorders: gastrointestinal symptoms (nausea, vomiting, diarrhoea) occur but disappear when the dose is reduced;

- nervous system disorders: dizziness, reduced blood pressure, seizures;

- immune system disorders: allergic dermatitis;

- eye disorders: impaired vision, blurred vision or colour vision impairment (chromatopsia);

- vascular disorders: thromboembolic events (acute myocardial infarction, thrombosis, arterial thrombosis limb, carotid artery thrombosis, cerebral infarction, cerebrovascular accident, deep vein thrombosis, pulmonary embolism, cerebral thrombosis, acute renal cortical necrosis and central retinal artery and vein obstruction).

*Information obtained from the Omega Laboratories product monograph for tranexamic acid injection (dated 30 August 2012). or renal failure with S-creatinine level $>250 \mu \mathrm{mol} / \mathrm{L} .^{28}$ The CRASH-2 trial that investigated the use of TXA in bleeding trauma patients showed a statistically significant reduction in global mortality with no increase in thromboembolic events. ${ }^{24}$ WOMAN trial, as discussed above, did not show that the use of TXA had any effect on the incidence of thromboembolic events, organ failure, sepsis or seizure in the more than 20000 patients studied. ${ }^{29}$

It should be noted that TXA crosses the placenta. However, after an intravenous injection of $10 \mathrm{mg} / \mathrm{kg}$ in the mother, the concentration only rises to a maximum of $31 \mu \mathrm{g} / \mathrm{mL}$ of foetal serum. ${ }^{30}$ Similarly, TXA also passes over into the breast milk during lactation in concentrations $1 / 100$ of the corresponding serum levels. ${ }^{30}$ Still, TXA is already commonly used in much higher doses among neonates and newborns who undergo immediate cardiac surgical procedures after birth-thus, there is some safety data on its use in these patients. In fact, a recent study examining the effects of pharmacokinetics of TXA in neonates (some even 2 days old) were given induction doses of $100 \mathrm{mg} / \mathrm{kg}$ doses of TXA intravenously followed by $10 \mathrm{mg} / \mathrm{kg} /$ hour throughout the surgery. ${ }^{31}$ Plasma concentrations were on average well beyond $200 \mu \mathrm{g} / \mathrm{mL}$ in such patients on average. ${ }^{31}$ This study, as with other studies on the use of TXA in such patients, have yet to report any increased risk of thrombosis or seizures related to the high dose use of TXA in newborns. ${ }^{31-33}$ However, the evidence supporting this routine use is considered weak. ${ }^{34}$ As a result, we have designed close monitoring for these events in our study in the newborn.

A prospective study conducted in women who continuously took oral TXA in the postpartum period demonstrated no increase in adverse long-term outcomes in infants exposed to TXA through breast feeding; suggesting a very low drug exposure and further supported the notation that women should continue breast feeding while taking TXA. ${ }^{35}$ It is highly unlikely that this conclusion will be any different among newborns who are exposed to breast milk from a mother who received only a single dose of TXA at delivery.

Finally, among three different recent systematic reviews on the prophylactic administration of TXA, there was no increase in major adverse events and/or venous or arterial thrombotic events detailed among both the mother in almost an aggregate of 2000 patients who received a prophylactic dose of TXA prior to delivery. ${ }^{322} 23$ Similarly, in the above systematic reviews, where in almost all cases TXA was given while the baby was in utero (prior to delivery), no obvious side effects were described in the newborns. Yet, reporting may have been suboptimal as these outcomes were not explicitly assessed. Thus, an important safety endpoint will be to closely monitor for major side effects in both the mother and the newborn both in the immediate and later postpartum periods.

\section{Ethical considerations}

All participants will be recruited in the antenatal period, prior to labour. They will receive detailed information 
regarding the study drug, its possible adverse effects and the possible effects on the fetus. Adverse events will be tracked in real time, and reporting will occur following institutional and Health Canada guidelines.

\section{Dissemination}

The trial data will be analysed and disseminated at scientific meetings, and in international peer-reviewed scientific journals. Safety and adverse event data will be reported to Health Canada where appropriate.

\section{DISCUSSION}

This pilot study will elucidate the feasibility for the prophylactic use of TXA for the prevention of PPH. It will also facilitate the development of a full-scale RCT that assesses the pragmatic utility and possible benefit for routine use of TXA in preventing PPH. Despite major resource investment in health system coordination and improved access to blood products in high-income settings, incidence of PPH continues to increase, resulting in significant maternal mortality and morbidity. Findings from WOMAN trial identified a simple, inexpensive, effective and safe intervention in decreasing mortality from PPH. The morbidity of $\mathrm{PPH}$, is nevertheless borne by parturients including requirement of blood product transfusion, operative haemostatic procedures and increased lengths of stay in hospital. TXA has been shown in the orthopaedic and cardiac elective surgery groups to be an effective, inexpensive preventative tool for blood product administration and subsequent morbidity associated with significant blood loss. Transformation of obstetric care and maternal health, particularly in mitigating the risks from PPH and blood loss in low-income, middle-income and high-income settings relies on prevention. TXA may play an important role in the continuing support of such a transformation.

This pilot study focuses on generalisability of results, and identifies the feasibility of TXA administration, along with providing important safety surveillance. The focus on management in high-resource settings is particularly imperative because of increasing incidence in the context of changing demographics of parturients. The study design also avoids sampling bias and measurement bias, by avoiding protocolised care.

Given the nature of the study, there are some challenges and limitations to data collection and reporting. The pilot study format is small, and the secondary outcomes have low incidences in general. As a result, clinical effect size will not be adequately captured in this pilot study. Furthermore, the intervention product delivery does not directly reflect normal current practice, and thus may not produce the same external validity. Finally, the setting that the trial is placed in a unique quaternary care centre with high-risk obstetrics, and represents a further stratification in high-resourced settings. Indeed, the feasibility data from this study may not be easily generalisable to lower-risk centres that do not have access to dedicated anaesthesiology, obstetrical and pharmacy supporthowever may allow for further study in which a more heterogeneous obstetrical care centres are involved.

The outcomes of a larger study can affect significant transformative change in maternal health and work towards meeting the redefined Millennium Development Goals of decreasing the maternal mortality ratio by three-quarters. The outcomes of WOMAN trial and its all-encompassing positive results for the usage of TXA underscores the importance of assessing safety and efficacy of an inexpensive, easily accessible medication in supporting women's health. In various parts of the world where heterogeneous practice environments exist, the effect of a large, generalisable study assessing the prophylactic use of TXA for safe obstetric care may have far reaching consequences on improving maternal haemorrhage.

\section{Author affiliations}

${ }^{1}$ Department of Anesthesia, Sunnybrook Health Sciences Centre, University of Toronto, Toronto, Ontario, Canada

${ }^{2}$ Trauma, Emergency and Critical Care Research Program, Sunnybrook Health Sciences Centre, University of Toronto, Toronto, Ontario, Canada

${ }^{3}$ Departmentof Obstetrics and Gynaecology, Sunnybrook Health Sciences Centre,

Univesity of Toronto, Toronto, Ontario, Canada

${ }^{4}$ Departmentof Clinical Pathology, Sunnybrook Health Sciences Centre, University of Toronto, Toronto, Ontario, Canada

${ }^{5}$ Evaluative Clinical Sciences, Hurvitz Brain Science Program, Sunnybrook Research Institute, Toronto, Ontario, Canada

Contributors AA conceived of the study question, hypothesis, carried out the evaluation of current literature, conceptualised the methodology and analysis, drafted and reviewed the manuscript. AB assisted with manuscript preparation. $\mathrm{SA}$ conceptualised the methodology and assisted in manuscript preparation. JB and $\mathrm{JC}$ assisted with the methodology and reviewed the manuscript. AK assisted in analysis and reviewed the manuscript. SC carried out the evaluation of current literation, conceptualised the methodology and reviewed the manuscript.

Funding This work is supported by the Sunnybrook AFP Innovation Fund. Competing interests None declared.

Patient consent None.

Ethics approval Sunnybrook Health Sciences Centre.

Provenance and peer review Not commissioned; externally peer reviewed.

Open Access This is an Open Access article distributed in accordance with the Creative Commons Attribution Non Commercial (CC BY-NC 4.0) license, which permits others to distribute, remix, adapt, build upon this work non-commercially, and license their derivative works on different terms, provided the original work is properly cited and the use is non-commercial. See: http://creativecommons.org/ licenses/by-nc/4.0/

(C) Article author(s) (or their employer(s) unless otherwise stated in the text of the article) 2017. All rights reserved. No commercial use is permitted unless otherwise expressly granted.

\section{REFERENCES}

1. American College of Obstetricians and Gynecologists. ACOG practice bulletin: clinical management guidelines for obstetriciangynecologists number 76, October 2006: postpartum hemorrhage. Obstet Gynecol 2006;108:1039-47.

2. Mpemba F, Kampo S, Zhang X, et al. Towards 2015: post-partum haemorrhage in sub-Saharan Africa still on the rise. J Clin Nurs 2014;23:774-83.

3. Alam A, Choi S. Prophylactic use of tranexamic acid for postpartum bleeding outcomes: a systematic review and meta-analysis of randomized controlled trials. Transfus Med Rev 2015;29:231-41. 
4. Carroli G, Cuesta C, Abalos E, et al. Epidemiology of postpartum haemorrhage: a systematic review. Best Pract Res Clin Obstet Gynaecol 2008;22:999-1012.

5. Calvert C, Thomas SL, Ronsmans C, et al. Identifying regional variation in the prevalence of postpartum haemorrhage: a systematic review and meta-analysis. PLoS One 2012;7:e41114.

6. Ford JB, Roberts CL, Simpson JM, et al. Increased postpartum hemorrhage rates in Australia. Int J Gynaecol Obstet 2007;98:237-43.

7. Joseph KS, Rouleau J, Kramer MS, et al. Investigation of an increase in postpartum haemorrhage in Canada. BJOG 2007;114:751-9.

8. Mehrabadi A, Hutcheon JA, Lee L, et al. Trends in postpartum hemorrhage from 2000 to 2009: a population-based study. BMC Pregnancy Childbirth 2012:12:108.

9. Mehrabadi A, Liu S, Bartholomew S, et al. Temporal trends in postpartum hemorrhage and severe postpartum hemorrhage in Canada from 2003 to 2010. J Obstet Gynaecol Can 2014;36:21-33.

10. Knight M, Callaghan WM, Berg C, et al. Trends in postpartum hemorrhage in high resource countries: a review and recommendations from the International Postpartum Hemorrhage Collaborative Group. BMC Pregnancy Childbirth 2009;9:55

11. Mehrabadi A, Hutcheon JA, Lee L, et al. Epidemiological investigation of a temporal increase in atonic postpartum haemorrhage: a population-based retrospective cohort study. BJOG 2013;120:853-62.

12. Irvine B, Dzakpasu S, Leon JA, et al. Perinatal health indicators 2013: a surveillance report by the Public Health Agency of Canada's Perinatal Surveillance System. Health Promot Chronic Dis Prev Can 2015;35:23-4.

13. Sentilhes L, Daniel V, Darsonval A, et al. Study protocol. TRAAP - TRAnexamic Acid for Preventing postpartum hemorrhage after vaginal delivery: a multicenter randomized, double-blind, placebocontrolled trial. BMC Pregnancy Childbirth 2015;15:5.

14. Pourat N, Martinez AE, McCullough J, et al. Costs of Maternal Hemorrhage in California, 2013.

15. Kirigia JM, Oluwole D, Mwabu GM, et al. Effects of maternal mortality on gross domestic product (GDP) in the WHO African region. Afr $J$ Health Sci 2006;13:86-95.

16. Hoylaerts M, Lijnen HR, Collen D. Studies on the mechanism of the antifibrinolytic action of tranexamic acid. Biochim Biophys Acta 1981:673:75-85.

17. Guerriero C, Cairns J, Perel P, et al. Cost-effectiveness analysis of administering tranexamic acid to bleeding trauma patients using evidence from the CRASH-2 trial. PLoS One 2011;6:e18987.

18. Ker K, Edwards P, Perel P, et al. Effect of tranexamic acid on surgical bleeding: systematic review and cumulative meta-analysis. $B M J$ 2012;344:e3054.

19. Perel P, Ker K, Morales Uribe $\mathrm{CH}$, et al. Tranexamic acid for reducing mortality in emergency and urgent surgery. Cochrane Database Syst Rev 2013:CD010245.

20. Elwatidy S, Jamjoom Z, Elgamal E, et al. Efficacy and safety of prophylactic large dose of tranexamic acid in spine surgery: a prospective, randomized, double-blind, placebo-controlled study. Spine 2008;33:2577-80.

21. Shakur H, Roberts I, Bautista R, et al. Effects of tranexamic acid on death, vascular occlusive events, and blood transfusion in trauma patients with significant haemorrhage (CRASH-2): a randomised, placebo-controlled trial. Lancet 2010;376:23-32.

22. Novikova N, Hofmeyr GJ, Cluver C. Tranexamic acid for preventing postpartum haemorrhage. Cochrane Database Syst Rev 2015:CD007872.

23. Simonazzi G, Bisulli M, Saccone G, et al. Tranexamic acid for preventing postpartum blood loss after cesarean delivery: a systematic review and meta-analysis of randomized controlled trials. Acta Obstet Gynecol Scand 2016;95:28-37.

24. Shakur H, Elbourne D, Gülmezoglu M, et al. The WOMAN Trial (World Maternal Antifibrinolytic Trial): tranexamic acid for the treatment of postpartum haemorrhage: an international randomised, double blind placebo controlled trial. Trials 2010;11:40.

25. Abdel-Aleem H, Alhusaini TK, Abdel-Aleem MA, et al. Effectiveness of tranexamic acid on blood loss in patients undergoing elective cesarean section: randomized clinical trial. J Matern Fetal Neonatal Med 2013;26:1705-9.

26. Yehia AH, Koleib MH, Abdelazim IA, et al. Tranexamic acid reduces blood loss during and after cesarean section: a double blinded, randomized, controlled trial. Asian Pacific Journal of Reproduction 2014;3:53-6.

27. Chan AW, Tetzlaff JM, Altman DG, et al. SPIRIT 2013 statement: defining standard protocol items for clinical trials. Ann Intern Med 2013;158:200-7.

28. Tan J, Chen H, Liu Q, et al. A meta-analysis of the effectiveness and safety of using tranexamic acid in primary unilateral total knee arthroplasty. J Surg Res 2013;184:880-7.

29. WOMAN Trial Collaborators. Effect of early tranexamic acid administration on mortality, hysterectomy, and other morbidities in women with post-partum haemorrhage (WOMAN): an international, randomised, double-blind, placebo-controlled trial. Lancet 2017;389.

30. Nilsson IM. Clinical pharmacology of aminocaproic and tranexamic acids. J Clin Pathol Suppl 1980;14:41-7.

31. Wesley MC, Pereira LM, Scharp LA, et al. Pharmacokinetics of tranexamic acid in neonates, infants, and children undergoing cardiac surgery with cardiopulmonary bypass. Anesthesiology 2015;122:746-58.

32. Grassin-Delyle S, Tremey B, Abe E, et al. Population pharmacokinetics of tranexamic acid in adults undergoing cardiac surgery with cardiopulmonary bypass. $\mathrm{Br} J$ Anaesth 2013:111:916-24.

33. Shimizu K, Toda Y, Iwasaki T, et al. Effect of tranexamic acid on blood loss in pediatric cardiac surgery: a randomized trial. $J$ Anesth 2011;25:823-30.

34. Faraoni D, Willems A, Melot C, et al. Efficacy of tranexamic acid in paediatric cardiac surgery: a systematic review and meta-analysis. Eur J Cardiothorac Surg 2012;42:781-6.

35. Gilad O, Merlob P, Stahl B, et al. Outcome following tranexamic acid exposure during breastfeeding. Breastfeed Med 2014;9:407-10. 